REVISTA MAD, Nº 35 (2016), PP. 38-59

\title{
Los usos del constructivismo en las publicaciones científicas de Latinoamérica
}

\author{
Uses of constructivism among Latin-American scientific publications
}

Gastón Becerra

Facultad de Filosofía y Letras, Universidad de Buenos Aires, Argentina

\begin{abstract}
RESUMEN
El constructivismo es un movimiento intelectual heterogéneo que cruza distintos campos del conocimiento. En su interior se registran diferentes discusiones que tratan con preguntas propias, referencias particulares, y que se concentran en las publicaciones especializadas de distintas disciplinas y áreas. En un intento por ordenar dicha comunicación, este trabajo revisa las publicaciones científicas de Latinoamérica que incluyen el término "constructivismo" entre sus campos, y que se encuentran indexados en las bases de datos CLASE, PERIODICA y SCIELO. Estas publicaciones son clasificadas en 3 grupos, de acuerdo al uso hacen del constructivismo: (1) los que buscan "aplicar" el constructivismo a los problemas de su campo; (2) los que toman desarrollos constructivistas como su "objeto" de estudio o crítica; (3) los que adoptan al constructivismo como un "marco" para análisis nocionales o conceptuales. Luego se describen comparativamente algunas características acerca de los 3 grupos de publicaciones (áreas de publicación, temas, keywords, principales autores) en un intento por observar cómo se organiza la comunicación científica en torno al constructivismo en Latinoamérica.
\end{abstract}

PALABRAS ClaVE: Publicaciones científicas latinoamericanas; Psicología y Educación; Epistemología; Cienciometría

\section{ABSTRACT}

Constructivism is a heterogeneous intellectual movement that spans across different fields of knowledge. Within constructivism there is a variety of discussions that deal with their own questions and particular references, and that appear clustered in the journals and publications of different scientific areas. Attempting to clarify this communication, the present paper explores scientific publications from Latin America that include the term "constructivism" among their descriptors, as listed on CLASE, PERIODICA and SCIELO databases. These publications have been segmented into 3 very general groups, according to the way in which constructivism is used: (1) those that seek to "apply" constructivism to the problems of their area; (2) those that take "constructivism" as their object of study or criticism; (3) those that adopt constructivism as a "framework" for notional or conceptual analysis. Some data about those publication groups is described and compared in an attempt to show how scientific communication about constructivism organizes in Latin America (publication area, subjects, keywords, main authors).

KEYWORDS: Constructivism; Scientific publications from Latin America; Psychology and Education; Epistemology; Science metrics

REVISTA MAD - REVISTA DEL MAGÍSTER EN ANÁLISIS SISTÉMICO APLICADO A LA SOCIEDAD

ISSN 0718-0527

Facultad de Ciencias Sociales, Departamento de Antropología.

Universidad de Chile

www.revistamad.uchile.cl

DOI: 10.5354/0718-0527.2016.42796 
Los usos del constructivismo en las publicaciones científicas de Latinoamérica

\section{INTRODUCCIÓN ${ }^{1}$}

El constructivismo es un movimiento heterogéneo que cruza varios campos del conocimiento científico, y que presenta distintas líneas de discusión en las que dialogan corrientes, conceptualizaciones, objetivos e intereses diversos. Esta generalidad ha conducido al diagnóstico por el cual el constructivismo "termina significando al mismo tiempo todo y nada" (Holstein \& Gubrium 2008: 5). Para ordenar esta situación en la literatura especializada se cuenta con varios trabajos que buscan esclarecer las diversas raíces, tesis y supuestos que circulan en los programas constructivistas en campos como la educación, la psicología, las ciencias sociales, la filosofía de las ciencias y la epistemología (Bickhard 1998; Castorina 2009; Delval 1997; Feixas Viaplana \& Villegas Besora 2000; Nola \& Irzik 2005; Sánchez 2009).

Pero la heterogeneidad del constructivismo no se agota en el plano de las tesis, los supuestos y las posiciones que subyacen a sus desarrollos. El constructivismo también es un campo de la comunicación científica que presenta diferencias en cuanto a los temas que se tratan y las áreas disciplinares para las que revisten alguna relevancia. Es en esta dimensión comunicativa que se centra el presente trabajo, y las distinciones teóricas que aquí presentamos no surgen de un análisis meta-teórico sino de una observación empírica sobre publicaciones científicas. Al centrarnos en la dimensión comunicativa, no negamos la heterogeneidad de posiciones y supuestos del constructivismo. Más bien buscamos observar que tales tensiones se enmarcan en una situación de fragmentación más amplia.

De esta forma, lo que aquí perseguimos no es otra cosa que una observación sociológica sobre un campo particular del sistema científico y la organización de sus desarrollos. En los términos de Niklas Luhmann (1997), las distinciones adoptadas persiguen una observación de segundo orden tendiente a dar cuenta de la diferenciación temática y disciplinar del constructivismo, en una forma muy elemental ya que sólo consideramos agrupamientos a partir de similitudes entre publicaciones, y no referencias y relaciones cruzadas entre distintas comunicaciones como se suele hacer en los análisis del "literary model" (Andersen 2003; Lucio-Arias \& Leydesdorff 2009). Aún con esta limitación, los agrupamientos aquí reseñados suponen una forma de autoorganización de las comunicaciones, ya que los artículos atraviesan un proceso de selección propio de las revistas de excelencia académica, en base a la relevancia que revisten en cuanto a enfoques, objetivos, marcos teóricos, lineamientos metodológicos y referencias bibliográficas para las distintas tradiciones y disciplinas (Becerra 2014; Stichweh 2008). Si estamos en lo cierto, deberíamos poder observar

\footnotetext{
${ }^{1}$ Artículo producido en el marco del proyecto «Investigaciones empíricas sobre el conocimiento de dominio social y sus implicaciones teórico-metodológicas». Director: José Antonio Castorina. Co-directora: Alicia Barreiro. Programa UBACYT 20020130100256BA, Facultad de Filosofía y Letras, Universidad de Buenos Aires (UBA).
} 
diferencias entre las características de distintos grupos de publicaciones. Por ello, aquí buscaremos rastrear estas diferencias a través de una descripción cienciométrica en torno a la distribución de temas, palabras claves (keywords), áreas de publicación y principales referencias a autores.

Nuestro corpus de trabajo incluye publicaciones científicas provenientes de Latinoamérica e indizadas en 3 bases de datos bibliográficas regionales: "CLASE" (Citas Latinoamericanas en Ciencias Sociales y Humanidades), "PERIODICA" (Índice de Revistas Latinoamericanas en Ciencias, más abocada a ciencias físicas y naturales), ambas creadas por la Universidad Nacional Autónoma de México en 1975, y "SCIELO" (Scientific Electronic Library Online), una iniciativa de UNESCO que cuenta con 15 años. Las 3 bases han sido seleccionadas por indizar sólo publicaciones de excelencia. Dicho corpus fue analizado con el objetivo de generar una clasificación teórica siguiendo laxamente los lineamientos metodológicos de la "grounded theory" (Glaser \& Strauss 1967), y las técnicas de análisis textual y de comparación constante. La compatibilidad de dicho enfoque con los análisis sistémicos ha sido sugerida por Gibson, Gregory y Robinson (2005).

Nuestra clasificación se centró en la presentación que las propias publicaciones hacen acerca del constructivismo, y cómo este se relaciona con los objetivos que asumen como propios. A falta de una mejor denominación, nos referiremos a distintos 'usos del constructivismo'. De nuestro análisis se desprenden 3 grupos de publicaciones que refieren a un uso particular: (A) los que toman al constructivismo 'como un enfoque general que se aplica' a los intereses de un campo particular; (B) los que toman al constructivismo como una corriente de pensamiento heterogénea compuesta por varios programas teóricos, de los cuales alguno o varios es adoptado como su 'objeto de estudio'; (C) los que adoptan al constructivismo como un 'marco analítico-metodológico' para el análisis crítico de conocimientos y nociones.

El trabajo se estructura de la siguiente forma: comenzamos con una breve sección metodológica, en la que reportamos el procedimiento con el que hemos construido el corpus de trabajo; luego, hacemos una caracterización del universo de las publicaciones acerca del constructivismo; finalmente, describimos los 3 grupos de publicaciones científicas en clave comparativa.

\section{MÉTODO}

Nuestro corpus de trabajo se confeccionó buscando el término "constructivismo" el 19 de agosto de 2015 en 3 bases de datos bibliográficas. Los resultados de dichas búsquedas se registraron en una planilla por medio de un proceso automatizado con macros de VBA. De los 686 registros devueltos originalmente, se eliminaron, a través de una revisión manual, más de 
Los usos del constructivismo en las publicaciones científicas de Latinoamérica

40 registros que tenían información duplicada o con la mayoría de sus campos faltantes. La caracterización de las publicaciones del constructivismo en América Latina se realizó en base a los 642 registros restantes. Luego, para la construcción de la categorización se trabajo sólo con aquellos 389 registros (aproximadamente el 60\% de la base de datos) que contaban con una URL para acceder al texto completo, insumo necesario para los análisis siguientes.

TABLA 1. BÚSQUEDA Y RESULTADOS EN BASES DE DATOS

\begin{tabular}{|c|c|c|c|}
\hline BASE & CAMPOS / FILTROS & $\begin{array}{c}\text { REGISTROS } \\
\text { (SIN DUPLICADOS) }\end{array}$ & $\begin{array}{l}\text { REGISTROS CON URL } \\
\text { A TEXTO COMPLETO }\end{array}$ \\
\hline CLASE & todos los campos / sin filtros & 496 & 267 \\
\hline PERIODICA & todos los campos / sin filtros & 51 & 12 \\
\hline SCIELO & todos los campos / sin filtros & 139 & 110 \\
\hline & TOTAL & 686 & 389 \\
\hline
\end{tabular}

Las 3 búsquedas devolvieron información acerca del título, autores, revista (nombre, año, número, volumen y páginas), y palabras claves (keywords). Para el análisis de estos datos fueron necesarios diferentes procedimientos de limpieza y normalización. Por ejemplo, en aquellos casos donde el año de publicación incluía un rango (ej.: 2014-2015), se dejó el último valor. Sólo en los keywords no se realizaron tareas de edición, de forma tal que errores ortográficos ("teoría" / "teoria") y términos no estandarizados ("Piaget" / "Piaget, Jean") cuentan como keywords separadas.

Los registros de las bases CLASE y PERIODICA incluían además información acerca de las 'áreas' de las revistas y los 'temas' de los artículos (podían ser más de uno), con referencia a 25 categorías muy similares aunque no idénticas. Para una mejor visualización de los datos, se agruparon algunas áreas por afinidad: revistas de comunicación, política, relaciones internacionales, epistemología de las ciencias sociales y sociología fueron agrupadas como "ciencias sociales y políticas"; revistas de pedagogía, educación, formación universitaria fueron agrupadas como "educación"; revistas de literatura, lingüística y arte fueron agrupadas bajo la etiqueta "literatura". Los registros provenientes de SCIELO no contaban con datos acerca de los "temas" de los artículos, de modo que para no excluirlos se adjudicó a cada artículo la información de las áreas de las revistas. En algunos registros, mayormente de CLASE y PERIODICA, se contó con otros datos como el país de la publicación, el idioma, o el tipo de publicación (artículo, ensayo, reseña bibliográfica, etc.) que no fueron considerados en nuestros análisis.

Como se mencionó, sólo los registros provenientes de SCIELO contaban con una URL para acceder al texto completo online. La información acerca de los autores más citados en las bibliografías se calculó sobre este subconjunto. De los 110 artículos con URL, sólo el 80\% tenían una 
bibliografía normalizada que se pudo importar para su procesamiento. El resultado fue una base de 2400 referencias bibliográficas repartidas en un total de 93 artículos. Dado que se trata de tan sólo un poco más que el 20\% de los registros clasificados, y menos del 15\% de la base total, estos resultados se deben considerar con mucho recaudo. Más aún, nuestro análisis se limitó a la frecuencia de citación de autores y no de obras. Así, por ejemplo, en lugar de contar por separado las veces que se citaron las obras "Psicología y pedagogía" y "Epistemología genética" de Jean Piaget simplemente se sumaron las referencias a "piaget j". Luego, para maximizar la visibilidad de los autores más citados, se optó por dejar sólo el primer autor de todas las referencias. Así por ejemplo, "Maturana, H. \& Varela, F." se contó solamente como "maturana h" (y este valor se sumó a las obras que Maturana escribió sin coautores). En el proceso de limpieza se borraron aquellos autores cuyas citas estaban contenidas en un solo artículo, y que en muchos casos, correspondía al mismo autor de la publicación analizada.

\section{EL CONSTRUCTIVISMO EN PUBLICACIONES CIENTÍFICAS DE AMÉRICA LATINA}

Como se observa en el gráfico 1 la indexación de artículos que tratan al constructivismo en sus diferentes formas prácticamente se duplicó en los últimos 10 años.

GRÁFICO 1. EVOLUCIÓN ANUAL DE ARTíCULOS INDEXADOS POR BASE DE DATOS BIBLIOGRÁFICA

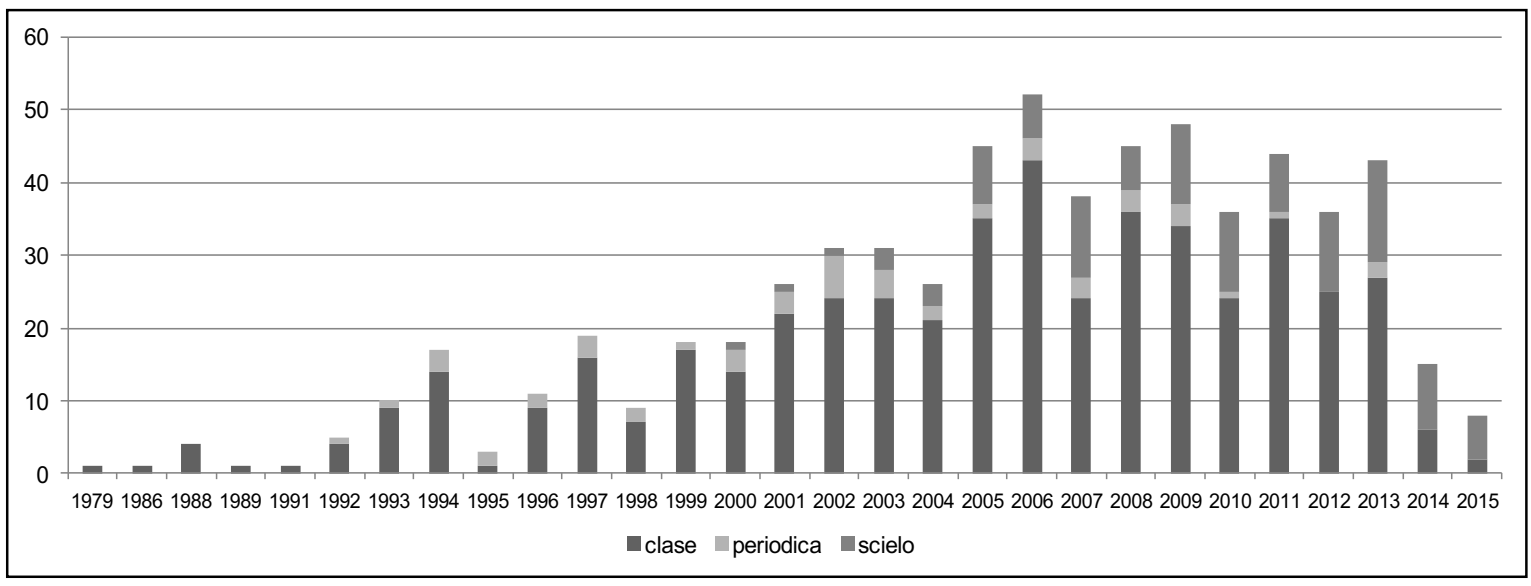

Los 642 artículos registrados corresponden a 364 revistas. La revista que concentra el mayor número de artículos indizados tiene 17 registros, mientras que la segunda tiene 8, y las que comparten el tercer lugar aportan 7 artículos. Estas primeras 9 revistas concentran aproximadamente el 10\% de los artículos de la base de datos.

La variedad de áreas de las 364 revistas puede ser tomado como un indicador de la heterogeneidad de campos en los que se inserta el constructivismo: administración, contaduría, antropología, arqueología, 
bibliotecología, ciencia de la información, biología, bioética, ciencia y tecnología, comunicación, política, relaciones internacionales, epistemología de las ciencias sociales y sociología, derecho, economía, ciencias de la educación, pedagogía, formación universitaria, filosofía, física, astronomía, geografía, urbanismo, historia, ingeniería, literatura, arte, lingüística, matemáticas, multidisciplinarias, psicología, química, medicina, enfermería y otras ciencias de la salud.

La distribución de publicaciones en estas áreas da cuenta de una cierta concentración. Como se puede observar en el gráfico 2, los primeros 5 campos que aportan más artículos son aquellos afines a la pedagogía (237 artículos en 92 revistas), las ciencias sociales y políticas (99 artículos en 62 revistas), la psicología (67 artículos en 38 revistas), los campos multidisciplinarios (70 artículos en 47 revistas), y a la filosofía (41 artículos en 28 revistas). Lo antedicho se mantiene en líneas generales, incluso si cambiamos la unidad del análisis, pasando del área de la revista al tema del artículo, tal como se observa en la tabla 2.

GRÁFICO 2. DiSTRIBUCIÓN DE ARTíCULOS Y REVISTAS POR ÁREA DE REVISTA

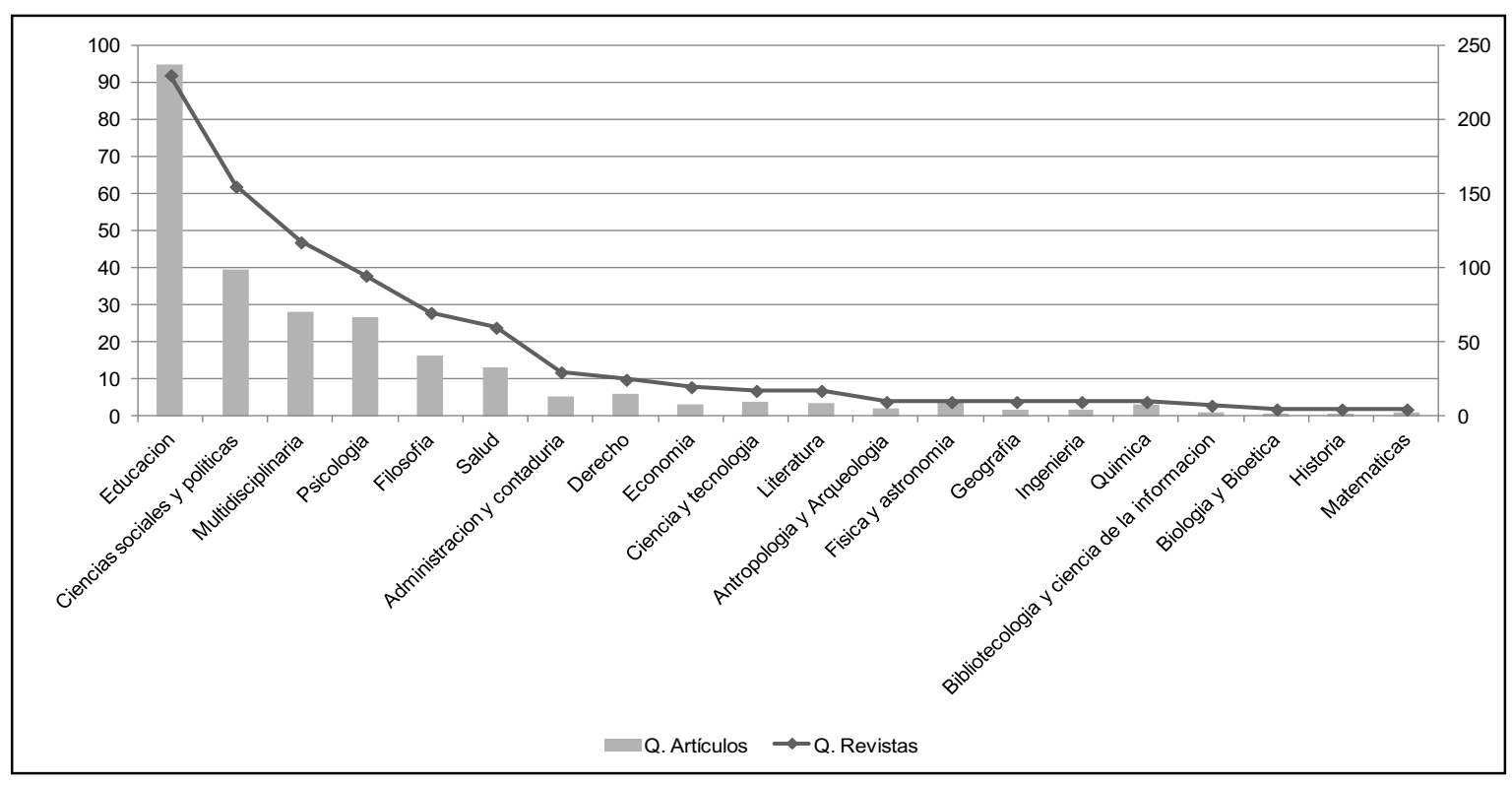

TABLA 2. DisTRIBUCIÓN DE TEMAS DE ARTíCULOS

\begin{tabular}{|c|c|c|c|c|c|}
\hline TEMAS & ARTí́CULOS & $\%$ ACUM. & TEMAS & ARTícULOS & $\%$ ACUM. \\
\hline Educación & 341 & $37,3 \%$ & Admin. y contaduría & 11 & $92,8 \%$ \\
\hline Psicología & 140 & $52,7 \%$ & Bibliotec. e información & 11 & $94,0 \%$ \\
\hline Cs. Sociales/polít. & 132 & $67,1 \%$ & Literatura y lingüística & 11 & $95,2 \%$ \\
\hline Filosofía & 93 & $77,3 \%$ & Física y astronomía & 9 & $96,2 \%$ \\
\hline Salud & 36 & $81,3 \%$ & Antrop. y arqueología & 7 & $96,9 \%$ \\
\hline $\begin{array}{c}\text { Ciencia y tecno- } \\
\text { logía }\end{array}$ & 24 & $83,9 \%$ & Ingeniería & 7 & $97,7 \%$ \\
\hline
\end{tabular}




\begin{tabular}{|c|c|c|c|c|c|}
\hline TEMAS & ARTícUlOS & $\%$ ACUM. & TEMAS & ARTíCULOS & $\%$ ACUM. \\
\hline Derecho & 20 & $86,1 \%$ & Química & 7 & $98,5 \%$ \\
\hline Matemáticas & 14 & $87,6 \%$ & Biología y bioética & 6 & $99,1 \%$ \\
\hline Arte & 12 & $88,9 \%$ & Geografía & 4 & $99,6 \%$ \\
\hline Economía & 12 & $90,3 \%$ & Multidisciplinaria & 2 & $99,8 \%$ \\
\hline Historia & 12 & $91,6 \%$ & Religión & 2 & $100,0 \%$ \\
\hline
\end{tabular}

Un indicador más fino - por estar menos normalizado- es el de las palabras claves o keywords de los artículos. En total se contaron 4.323 keywords, con 1.600 valores únicos (sin contar valores repetidos). Los primeros 16 keywords más mencionadas concentran el 30\% de la distribución total. Con la excepción obvia del keyword "constructivismo" (478 repeticiones), estos se encuentran fuertemente vinculados a los temas mencionados: pedagogía (168), aprendizaje y enseñanza (sumando también a cada término por separado) (165), didáctica (85), psicología educativa (65), educación superior (56), historia y filosofía de la educación (43), epistemología (39), conocimiento (32), desarrollo psicológico (30), investigación educativa (30), constructivismo social (28), evaluación educativa (26), gnoseología (25), historia y teoría de la sociología (25).

Para el cálculo de los autores más citados en la bibliografía de los artículos se analizaron más de 2400 referencias, con aproximadamente 1700 valores únicos, de los cuales sólo 139 fueron referenciados más de 3 veces. Los autores más referenciados se listan la tabla 3 (el orden de los autores en la tabla corresponde a la cantidad de artículos que referencian al autor, y no a la cantidad de referencias totales, con el fin de evitar sobredimensionar a autores con muchas referencias en pocos trabajos). Este es otro indicador de la heterogeneidad -ahora de fuentes- del constructivismo, que incluyen tanto a referentes clásicos de la filosofía y la epistemología como a autores actuales de investigación en campos y corrientes mencionadas.

TABLA 3. AUTORES MÁS CITADOS EN LAS REFERENCIAS BIBLIOGRÁFICAS

\begin{tabular}{|c|c|c|c|c|c|}
\hline AUTOR & ARTíCULOS & REFERENCIAS & AUTOR & ARTíCULOS & REFERENCIAS \\
\hline Vygotsky ls & 19 & 25 & Hacking $\mathrm{i}$ & 4 & 5 \\
\hline Piaget j & 15 & 20 & Boix mansilla v & 4 & 4 \\
\hline Maturana $\mathrm{h}$ & 7 & 19 & Kuhn $\mathrm{t}$ & 4 & 4 \\
\hline Freire $\mathrm{p}$ & 7 & 13 & Savery j & 4 & 4 \\
\hline Ausubel $\mathrm{d}$ & 7 & 8 & Kant $\mathrm{i}$ & 3 & 12 \\
\hline Carretero $\mathrm{m}$ & 7 & 7 & Pozo j & 3 & 9 \\
\hline Rawls j & 6 & 16 & Fodor j & 3 & 7 \\
\hline Perrenoud $\mathrm{p}$ & 6 & 15 & Ferreiro e & 3 & 6 \\
\hline Coll c & 6 & 14 & Bruner j & 3 & 4 \\
\hline Dewey j & 6 & 11 & Bardin $~$ & 3 & 3 \\
\hline Bourdieu $\mathrm{p}$ & 6 & 6 & Bateson $\mathrm{g}$ & 3 & 3 \\
\hline Foucault $\mathrm{m}$ & 6 & 6 & Bruffee $\mathrm{k}$ & 3 & 3 \\
\hline Luhmann $\mathrm{n}$ & 6 & 6 & Chomsky $\mathrm{n}$ & 3 & 3 \\
\hline
\end{tabular}


Los usos del constructivismo en las publicaciones científicas de Latinoamérica

\begin{tabular}{|c|c|c|c|c|c|}
\hline AUTOR & ARTÍCULOS & REFERENCIAS & AUTOR & ARTÍCULOS & REFERENCIAS \\
\hline Morin $\mathrm{e}$ & 6 & 6 & Gonzalez $\mathrm{f}$ & 3 & 3 \\
\hline Castorina ja & 5 & 13 & Oliveira mk & 3 & 3 \\
\hline Perkins d & 5 & 7 & Ordonez c & 3 & 3 \\
\hline Berger $\mathrm{p}$ & 5 & 5 & Popper $\mathrm{k}$ & 3 & 3 \\
\hline Habermas $j$ & 5 & 5 & UNESCO & 3 & 3 \\
\hline Latour b & 4 & 17 & Venturelli $j$ & 3 & 3 \\
\hline Wendt a & 4 & 12 & Weber $\mathrm{m}$ & 3 & 3 \\
\hline Skinner b & 4 & 7 & & & \\
\hline
\end{tabular}

3. LOS USOS DEL “CONSTRUCTIVISMO” EN LAS COMUNICACIONES CIENTÍFICAS DE AMÉRICA LATINA

En la sección precedente se buscó dar con algunos indicadores que pudieran servirnos de 'primer pantallazo' sobre cómo se estructura la comunicación en torno al constructivismo en las publicaciones científicas de América Latina. En lo que sigue, construimos 3 grupos de artículos en torno a un 'uso del constructivismo' particular, que conforman un recorte de las comunicaciones previamente analizadas.

TABLA 4. DisTRIBUCIÓN DE PUBLICACIONES DE ACUERDO AL USO DEL CONSTRUCTIVISMO

\begin{tabular}{|c|c|c|c|}
\hline GRUPO & USO DEL CONSTRUCTIVISMO & ARTÍCULOS & $\%$ ACUM. \\
\hline A & La aplicación del constructivismo & 219 & $56 \%$ \\
\hline B & El constructivismo como objeto & 87 & $79 \%$ \\
\hline C & El constructivismo como un marco & 83 & $100 \%$ \\
\hline \multicolumn{2}{r}{ TOTAL } & 389 & \\
\hline
\end{tabular}

Esta clasificación se realizó analizando los textos completos de los artículos. Si bien el criterio utilizado para la construcción de estos grupos fue el de la saturación teórica, hemos buscamos reducir la cantidad de grupos construidos para contar con suficientes artículos como para analizar variaciones en los indicadores de las publicaciones. Además, para maximizar la visibilidad de estas diferencias se optó por clasificar los artículos en forma excluyente. Esta decisión metodológica es cuestionable porque ni los usos dados ni las formas de discutir al constructivismo son unívocos.

\subsection{GRUPO "A": PUBLICACIONES QUE APLICAN PROGRAMAS CONSTRUCTIVISTAS}

El primer grupo que se delimitó corresponde a los artículos que utilizan enfoques constructivistas para actuar sobre las problemáticas particulares de algún campo. Mayormente este grupo incluye artículos cuyo objetivo es reportar, investigar o reflexionar sobre enseñanza general o especializada, y en menor medida algunos artículos de clínica y terapia psicológica de corte constructivista. Entre los temas más tratados se incluyen: el análisis de experiencias didácticas en distintos niveles educativos y en distintos 
campos del conocimiento, así como el estudio de caso y comparación de distintos modelos y sistemas educativos, la implementación de nuevas tecnologías en las prácticas docentes, la discusión de problemas de currículo y evaluación, y reflexiones generales sobre el impacto del constructivismo como una corriente de pensamiento para los problemas de la educación y la enseñanza.

Más de la mitad de los artículos de este grupo (aproximadamente $51 \%$ ) se encuentran publicados en revistas afines a la de educación (Gráfico 3). Sin embargo temáticas como las mencionadas cruzan prácticamente todo el espectro de áreas de revistas relevadas: así, por ejemplo, revistas de salud (aproximadamente $8 \%$ ) refieren a problemas propios de la enseñanza de la enfermería y la clínica; revistas del área de las ciencias sociales y psicología (aproximadamente 8\% y 10\% respectivamente) incluyen artículos sobre la relación entre educación y promoción de prácticas en distintos grupos sociales, o de los cambios y desafíos en los roles de los docentes y estudiantes en relación a problemas psicosociales.

Se observan datos semejantes si pasamos del área de las revistas hacia los temas de los artículos (Gráfico 4). De nuevo aquí los principales temas son: educación $(54 \%)$, psicología $(14 \%)$, ciencias sociales y políticas (7\%) y salud (6\%). Luego, si observamos los keywords de los artículos (Gráfico 5) vemos también un predominio del campo semántico de la pedagogía: de las 670 keywords analizadas, las primeras 5 (constructivismo, enseñanza y aprendizaje, pedagogía, didáctica, y educación superior) suman más del $60 \%$ sobre el total.

Los datos anteriores nos permiten hablar de un grupo con comunicaciones concentradas. De ninguna forma esto implica que el constructivismo aquí tratado sea una corriente homogénea, ni menos un único desarrollo teórico consistente. Como señalan varios autores, en estos campos el constructivismo es un conglomerado heterogéneo que comparte sólo lineamientos muy básicos (Castorina 2009; Coll 1996; Delval 2001).

Esta heterogeneidad se puede observar listando los autores clásicos más referenciados en este grupo (Gráfico 6): Lev Vigotsky, Jean Piaget, Philippe Perrenoud y David Ausubel. Luego siguen otras referencias a académicos contemporáneos (en algunos casos estudiosos o críticos de los primeros) también insertos en tradiciones diversas, como Cesar Coll, Paulo Freire, David Perkins, Juan Ignacio Pozo y Mario Carretero. La lista también incluye a otros autores de epistemología, y de las disciplinas particulares, tales como Humberto Maturana, James Dewey, Pierre Bourdieu, Michel Foucault, o Edgar Morin. Esta configuración que combina concentración con heterogeneidad también ha sido observada en otro trabajo de revisión sobre publicaciones latinoamericanas de psicología y educación (Castellaro 2012). 
GRÁFICO 3. DiSTRIBUCIÓN DE ARTÍCULOS POR ÁREA DE REVISTA

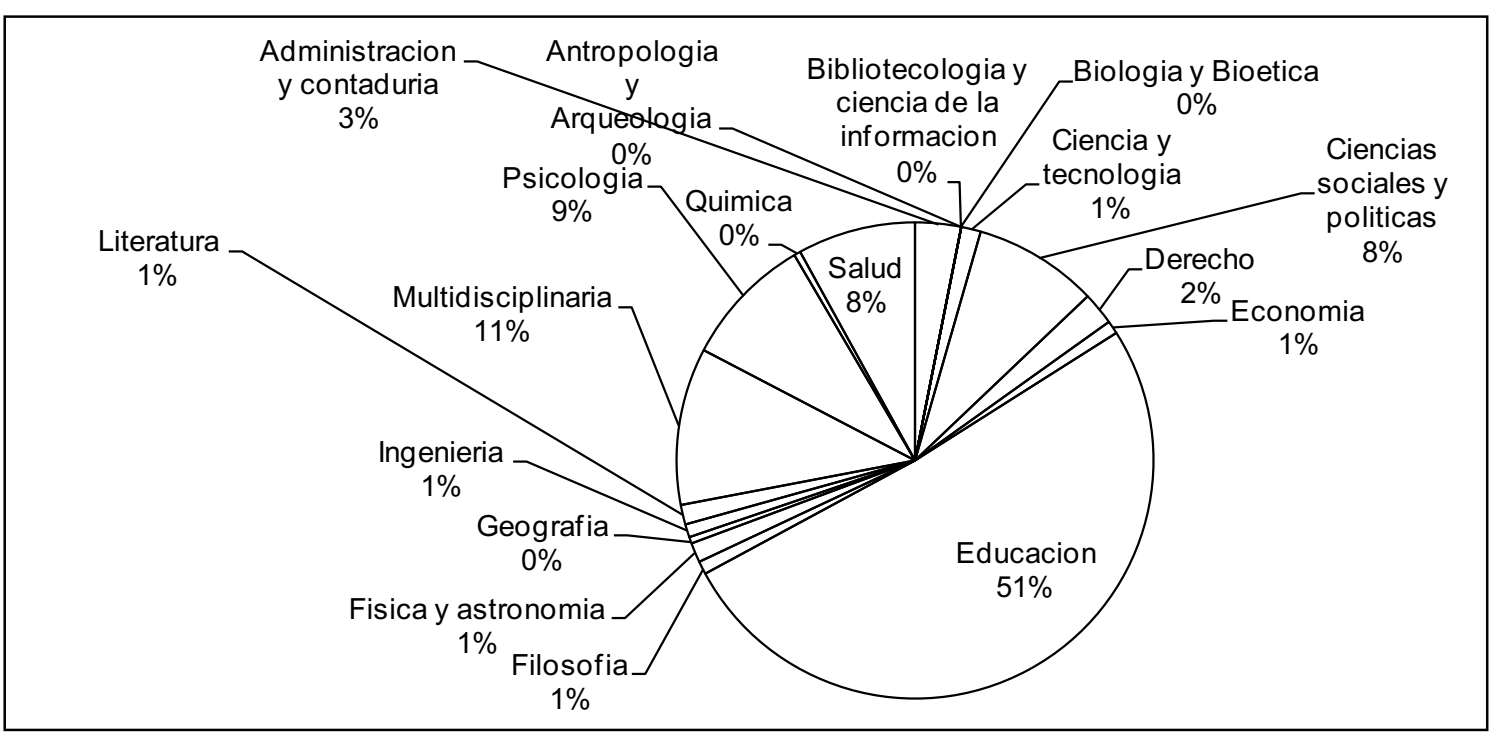

GRÁFICO 4. DiSTRIBUCIÓN DE ARTíCULOS POR TEMA DE ARTÍCULO

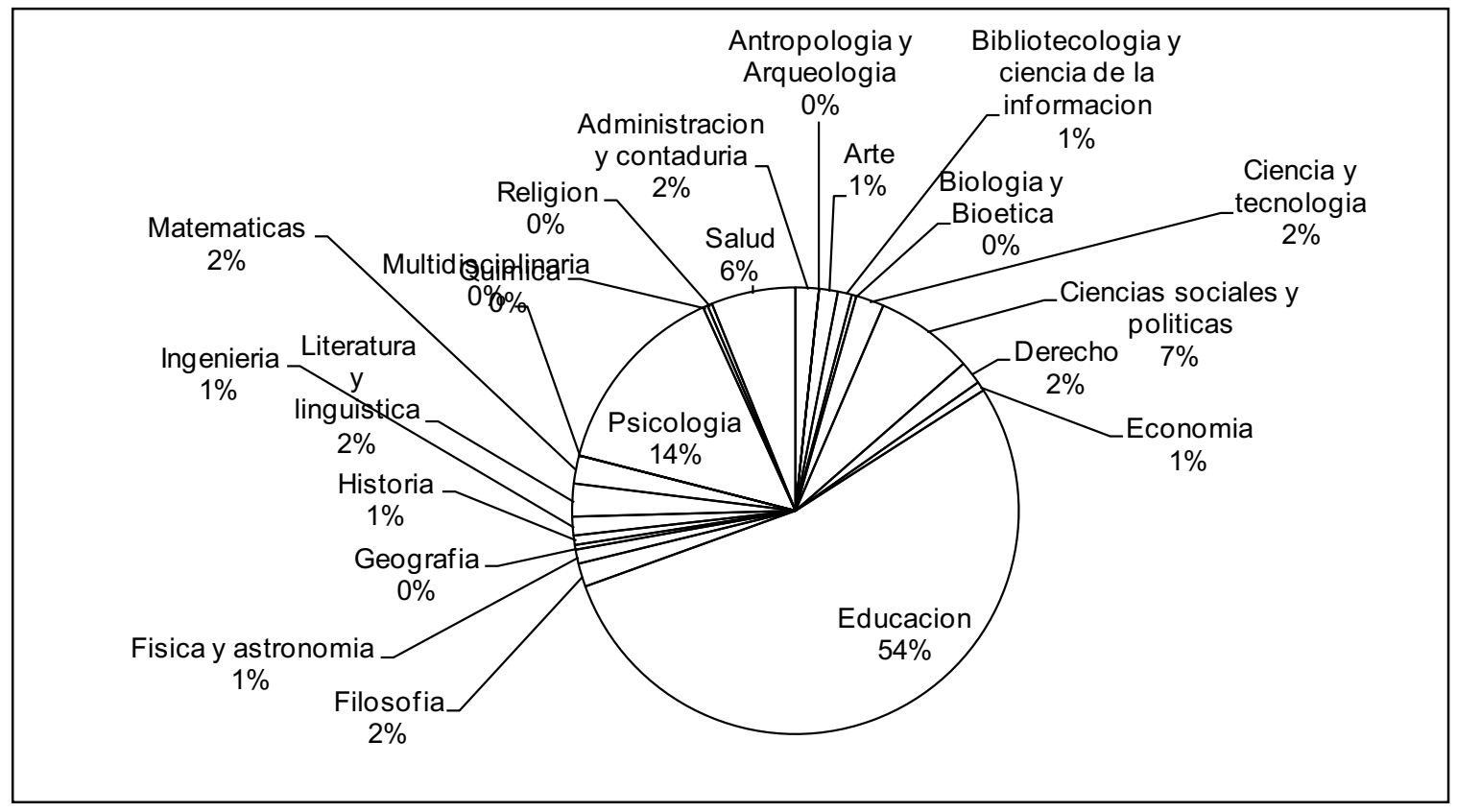




\section{GRÁFICO 5. DiSTRIBUCIÓN DE KEYWORDS}

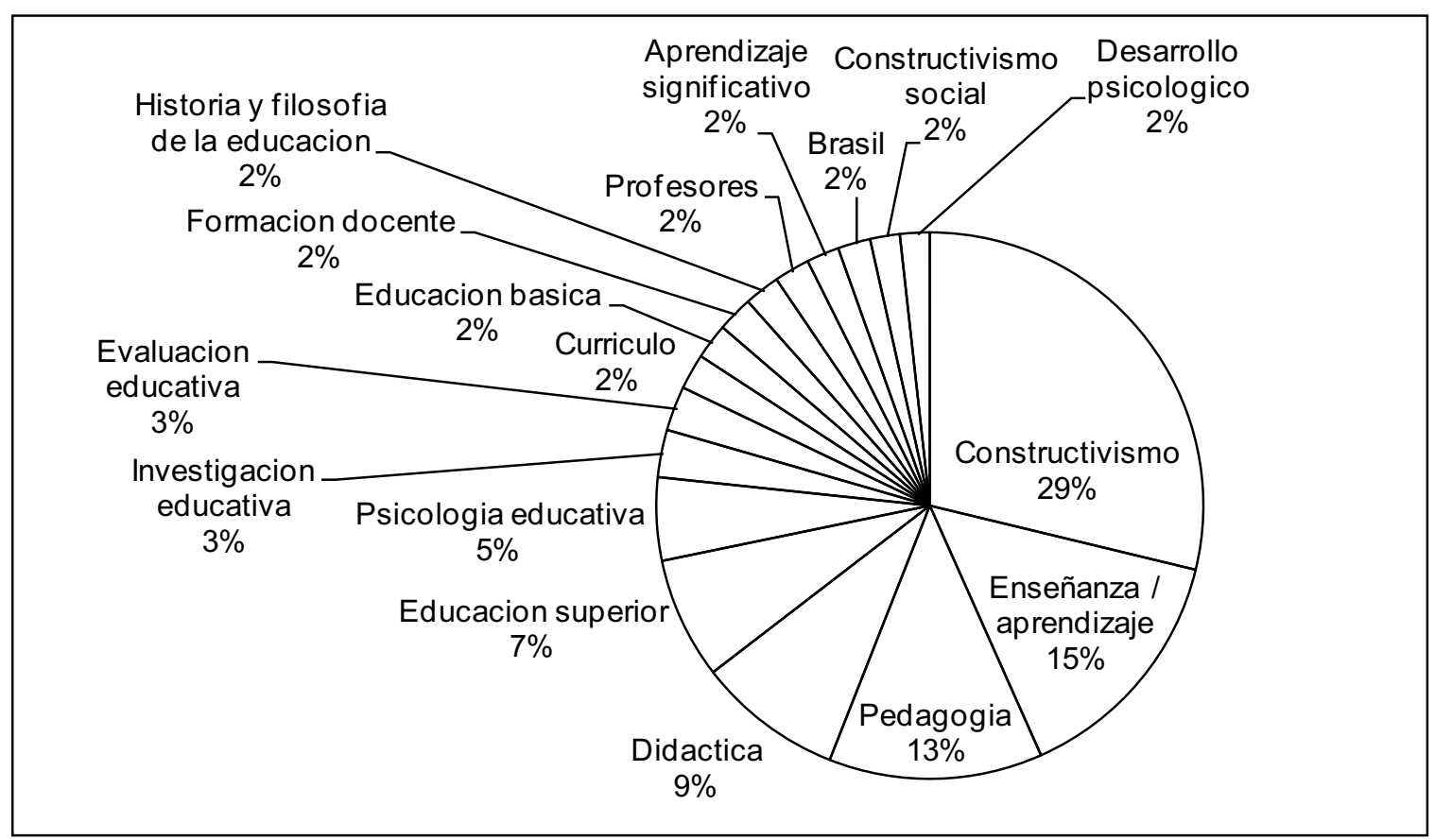

\section{GRÁFICO 6. REFERENCIA A AUTORES}

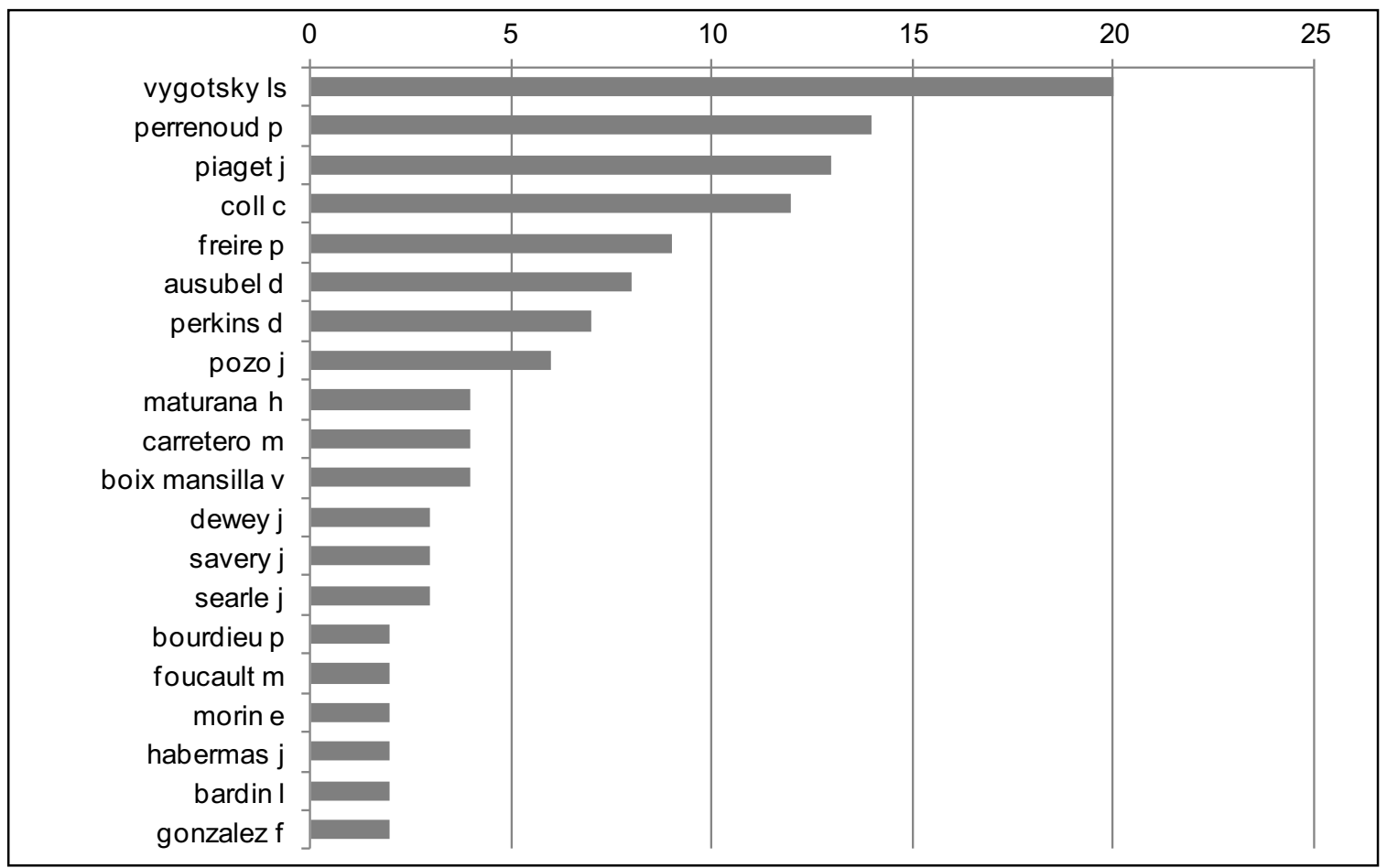


Los usos del constructivismo en las publicaciones científicas de Latinoamérica

\subsection{GRUPO “B”: EL CONSTRUCTIVISMO COMO UN OBJETO DE ESTUDIO}

El segundo grupo que hemos delimitado se caracteriza por tomar a los distintos programas constructivistas (teorías, tradiciones, proyectos de investigación) como su objeto de estudio. El enfoque predominante en este grupo es el del análisis conceptual o "meta-teórico" (Castorina 2007). Generalmente su objetivo es indagar y aclarar los supuestos de las teorías, lo que ubica a las publicaciones en el campo de las filosofías y epistemologías especiales de los campos disciplinares.

En estas publicaciones, cuando se habla de constructivismo se refiere a desarrollos particulares: constructivismo kantiano, luhmanniano, latouriano, radical, biológico, etc. Es muy común que estos desarrollos se vean como la expresión del constructivismo al interior de un campo disciplinar acotado. Por ello, los artículos de este grupo suelen advertir la necesidad de pensar tales desarrollos en diálogo con otros programas constructivistas, enfrentando los supuestos subyacentes y finalmente complejizando la imagen del constructivismo.

Un caso en el que se observa esto claramente es el de los artículos que discuten los fundamentos teóricos y epistemológicos del 'constructivismo pedagógico'. Es decir, la reflexión sobre la heterogeneidad del 'constructivismo aplicado' del grupo A queda considerada en este grupo B. La distinción es útil porque permite visualizar otros indicadores que se hubieran diluido en la mayor concentración del primer grupo. Aquí los referentes en la filosofía de la educación y la pedagogía suelen advertir a los investigadores y profesionales del área sobre las incompatibilidades de las tesis de programas constructivistas tan diversos como la epistemología genética de Piaget, el enfoque radical de Glasersfeld, el social de Gergen, de la psicología cognitiva, de las biologías del aprendizaje y la cibernética, o de la teoría sociocultural del aprendizaje de Vygotsky. Así, por ejemplo, en un artículo ampliamente citado, Cesar Coll reflexiona sobre la unidad del constructivismo pedagógico informado por tales fuentes, y afirma: "Basta revisar las explicaciones de los procesos escolares de enseñanza y aprendizaje, o aun las aplicaciones concretas a la educación escolar que se proponen desde cada una de ellas, para caer en la cuenta de que, en efecto, no tiene mucho sentido hablar de constructivismo en general y si tal vez de distintos tipos de constructivismos" (Coll 1996: 155). En el mismo sentido, Castorina (2009) advierte que varios trabajos en educación o psicología que refieren al "constructivismo" lo hacen adoptando un programa particular constructivista, sin observar que el alcance de sus tesis y estrategias metodológicas se comprende mejor en la comparación entre programas alternativos.

En comparación con las comunicaciones del grupo A, aquí se observa una cierta dispersión disciplinar. De los aproximadamente 80 artículos revisados, las 5 primeras áreas de revistas comparten prácticamente la misma proporción: filosofía (21\%), ciencias sociales y políticas $(19 \%)$, 
educación (19\%), psicología (14\%) y multidisciplinaria (13\%). Las primeras 4 son también los temas predominantes de los artículos (Gráfico 8), aunque esta coincidencia bien puede ser producto de nuestra decisión metodológica de adjudicar las áreas a los temas en los registros que no contaban con esta información. El dato sólo se vuelve valioso si lo vemos en clave comparativa con los demás grupos: aquí observamos un leve incremento de la incidencia de los artículos catalogados como 'filosóficos' (aproximadamente al 29\%), incluso cuando el objeto de estudio son corrientes constructivistas de la sociología o la psicología -por ejemplo, los estudios sobre la obra de Niklas Luhmann o de Jean Piaget-, lo que puede interpretarse como un indicador de que aquí 'filosofía' refiere más al enfoque que al objeto.

En lo que respecta a los keywords (Gráfico 9), es fácil constatar una disminución relativa de la presencia de aquellos términos asociados a la pedagogía y la educación, y en su lugar, un aumento de términos que ubican al constructivismo en un área específica de discusión disciplinar (doctrinas y corrientes filosóficas; ética; pedagogía; psicología social y educativa; historia y filosofía de la psicología y la sociología, el derecho, etc.). Algo similar podríamos decir de los autores más referidos, mayormente clásicos y contemporáneos, exponentes de formas de constructivismo en distintas disciplinas: Immanuel Kant, John Rawls, Humberto Maturana, John Dewey o Kenneth Gergen.

GRÁFICO 7. DisTRIBUCIÓN DE ARTÍCULOS POR ÁREA DE REVISTA

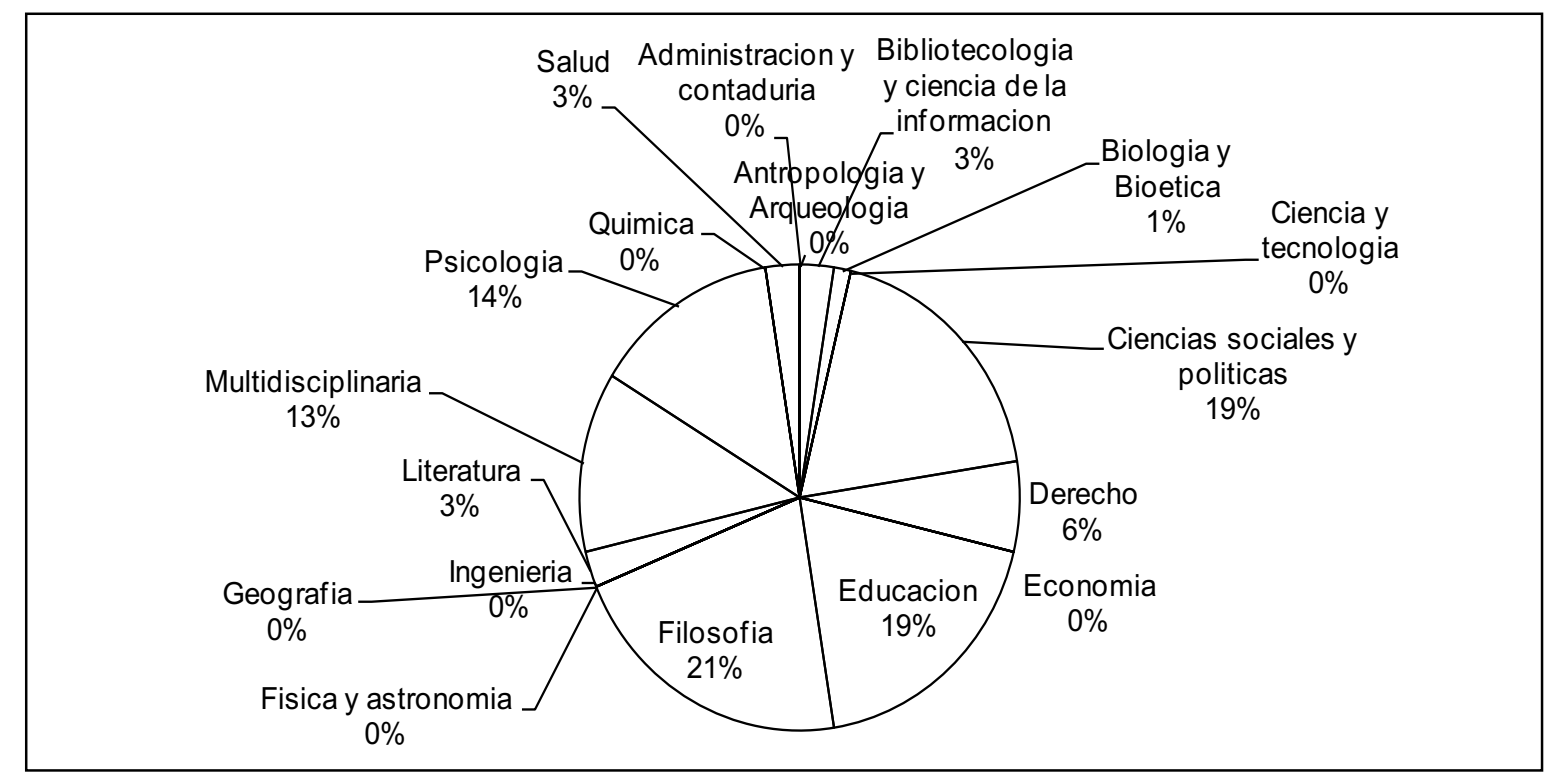




\section{GRÁFICO 8. DiSTRIBUCIÓN DE ARTÍCULOS POR TEMA}

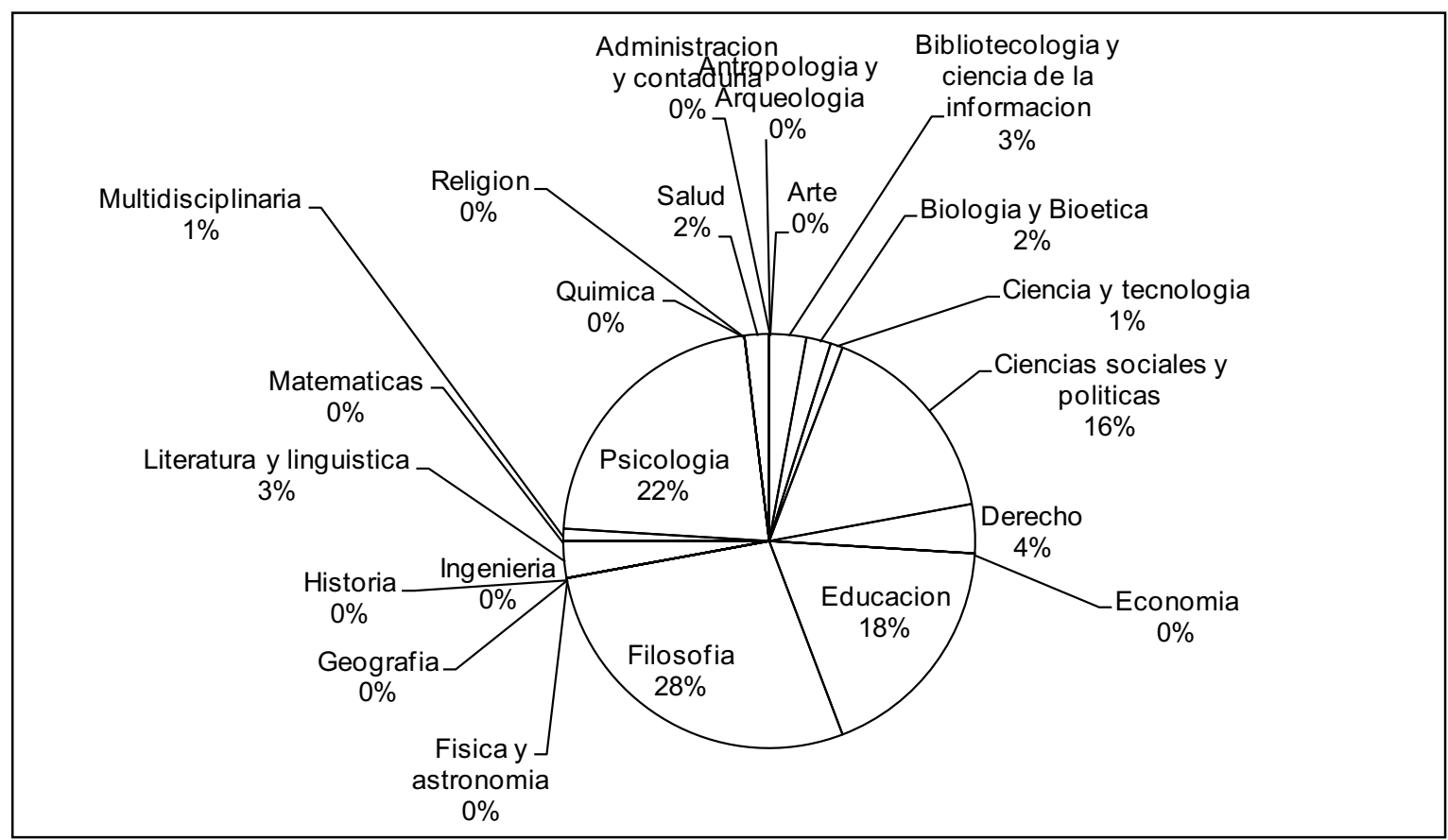

GRÁFICO 9. DiSTRIBUCIÓN DE KEYWORDS

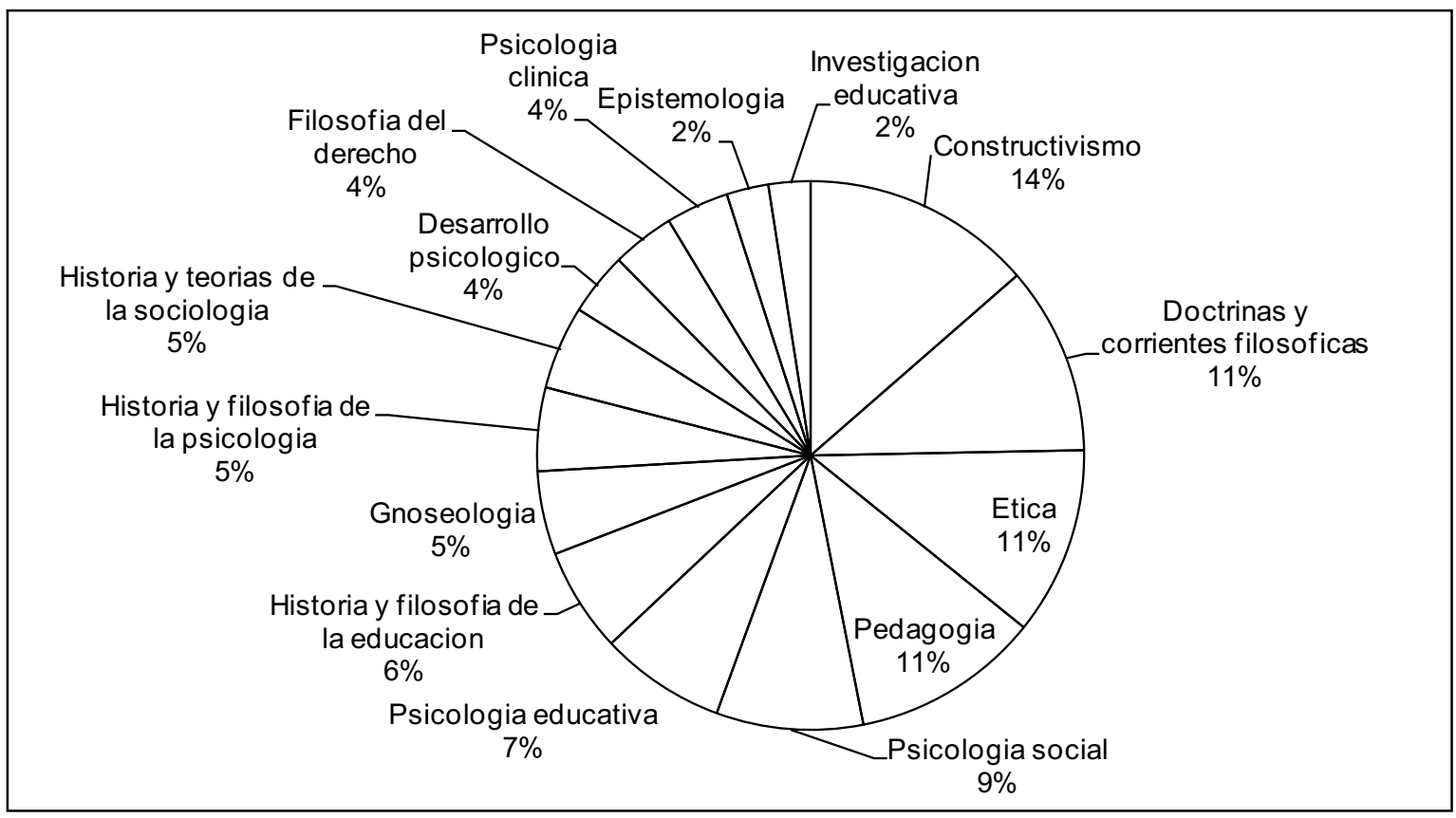


GRÁFICO 10. REFERENCIAS A AUTORES

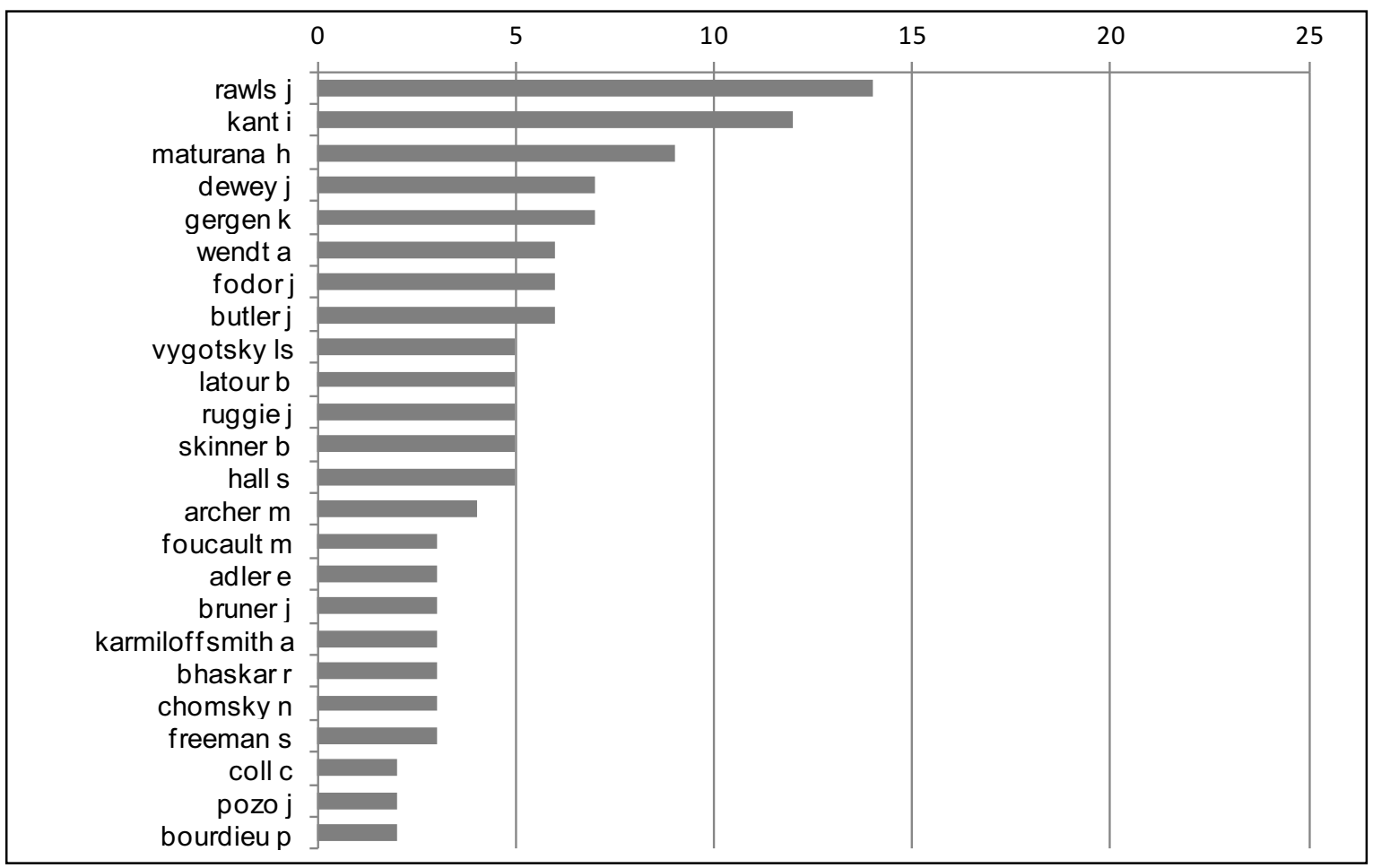

3.3. Grupo "C": el CONSTRUCTIVISMO COMO UN ENFOQUE CRÍtico SOBRE CONOCIMIENTOS Y NOCIONES

El tercer grupo que hemos delimitado está constituido por aquellos artículos, cuyo objeto de análisis es la construcción de ciertas nociones y conocimientos. Este sentido del constructivismo -sin duda, el más vaporoso- es similar a los implicados en los otros dos grupos, por lo que conviene aclararlo distinguiendo dos subgrupos. Sin embargo, debemos señalar que como este tercer grupo es el que contiene menos artículos (aproximadamente un 20\% del subconjunto analizado), no se cuenta con casos suficientes como para calcular los observables en términos de esta distinción sino que allí volveremos a tratarlos como un único grupo.

En primer lugar, observamos un subconjunto de artículos que refieren a la construcción de conocimientos y nociones generales, pero sin restringirse a los conocimientos que importan a la práctica educativa o clínica como era característico del grupo A. Más bien, se trata de trabajos sobre la construcción de nociones y representaciones sociales, y hasta de significados y símbolos culturales. Muchos de estos trabajos parten de una "actitud" que niega la naturalidad o la inevitabilidad con la que se presentan dichas nociones en la vida social (Hacking 1999: 12; Kukla 2000: 3).

Varias disciplinas toman parte de esta tarea, desde la psicología del desarrollo y la psicología social -ejemplo de este tipo de artículos incluyen a la construcción de conceptualizaciones infantiles e institucionales sobre 
Los usos del constructivismo en las publicaciones científicas de Latinoamérica

nociones sociales tales como la privacidad y la intimidad, la propiedad y el robo, la autoridad, entre otros; o sobre fenómenos sociales como la producción de noticias o el funcionamiento de internet-, hasta la sociología, los estudios políticos y de relaciones internacionales, la antropología y los estudios culturales -ejemplos de este tipo de artículos son aquellos que tratan sobre la construcción discursiva de clasificaciones sociales cómo el género o las edades, o de instituciones sociales tales como países y naciones, derechos humanos, etc.-. Cada uno de estos enfoques introduce sus propias preguntas, métodos y unidades de análisis, y hasta una conceptualización diferente que se ha plasmado en la distinción entre programas "constructivistas" y "construccionistas (sociales)" (Guzzini 2000; Munné 1999; Noss \& Clayson 2009).

En segundo lugar, se pueden mencionar otras publicaciones, cuyo objeto de estudio son los conocimientos vinculados a teorías y desarrollos científicos. Lo que diferencia a estos artículos de los mencionados en el grupo B es que no se restringen a programas constructivistas, sino a teorizaciones y enfoques dominantes en una rama del conocimiento. Así, el sentido particular que el constructivismo adquiere en este grupo es el de un 'marco metateórico, epistemológico o crítico'. Más aún, en muchos casos estos artículos explícitamente asumen que su objeto de estudio son "paradigmas" en el sentido de Kuhn (1970), o al menos, corrientes muy influyentes en dichos campos. Un caso de este tipo de análisis constructivista, con pocas referencias en la muestra, pero con una reflexión muy clara sobre lo que queda implicado, ha sido desarrollado por Rolando García en torno a la noción de "marco epistémico", fuertemente inspirada por la "epistemología interna" de Jean Piaget: los marcos epistémicos son supuestos que operan en la concepción científica de un programa de investigación o de una rama del conocimiento y que condicionan desde la problematización que se hace del mundo hasta las conceptualizaciones y las decisiones metodológicas (García 2000; Becerra \& Castorina 2016). Entre los campos que se discuten desde esta visión constructivista se destacan los estudios sobre movimientos sociales, las problemáticas ecológicas y ambientales, la administración, y las teorías psicológicas.

Volviendo ahora a los datos con los que podemos caracterizar a este grupo de publicaciones $\mathrm{C}$, se puede constatar que el área de la revista predominante (Gráfico 11) es el de las ciencias sociales (44\%), con un porcentaje casi tan alto como el que tenía el área de educación en el primer grupo. Le siguen los trabajos publicados en revistas de educación (9\%) y estudios multidisciplinarios (9\%). Algo similar se puede decir en relación a los temas de los artículos (Gráfico 12), donde a las ciencias sociales y políticas $(42 \%)$ les siguen por una diferencia análoga los artículos de educación (13\%), filosofía (8\%) y psicología (8\%).

El análisis de keywords se ve condicionado por un subconjunto muy pequeño de registros y una gran dispersión en comparación con los grupos anteriores (se registraron 110 keywords, cuyas primeras 5 entradas más 
repetidas no suman el 25\% del total). Sin embargo, se debe destacar que este tercer grupo es el único en el que no se prioriza el término constructivismo. Su particularidad se refleja en una mayor incidencia de campos de estudios relativos a la historia, la filosofía, la teoría y hasta la sociología de distintas disciplinas como la sociología (9\%), las relaciones internacionales $(6 \%)$, educación (5\%), o filosofía (4\%).

Los dos subgrupos aquí referidos se hacen visibles en los autores más citados (Gráfico 13). Por un lado, se incluyen investigadores contemporáneos de los enfoques constructivistas y construccionistas -José A. Castorina, Flora Perelman, Daniel Hiernaux-, que conviven en las publicaciones con referentes como Jean Piaget, Humberto Maturana, Bruno Latour, Niklas Luhmann, Berger \& Luckmann. Por su parte, el segundo subgrupo aquí mencionado introduce varios autores de que abrevan en la filosofía de las ciencias contemporáneas desde tradiciones muy diversas: Thomas Kuhn, Karl Popper, Mario Bunge o Edgar Morin.

GRÁFICO 11. DisTRIBUCIÓN DE ARTíCULOS POR ÁREA DE REVISTA

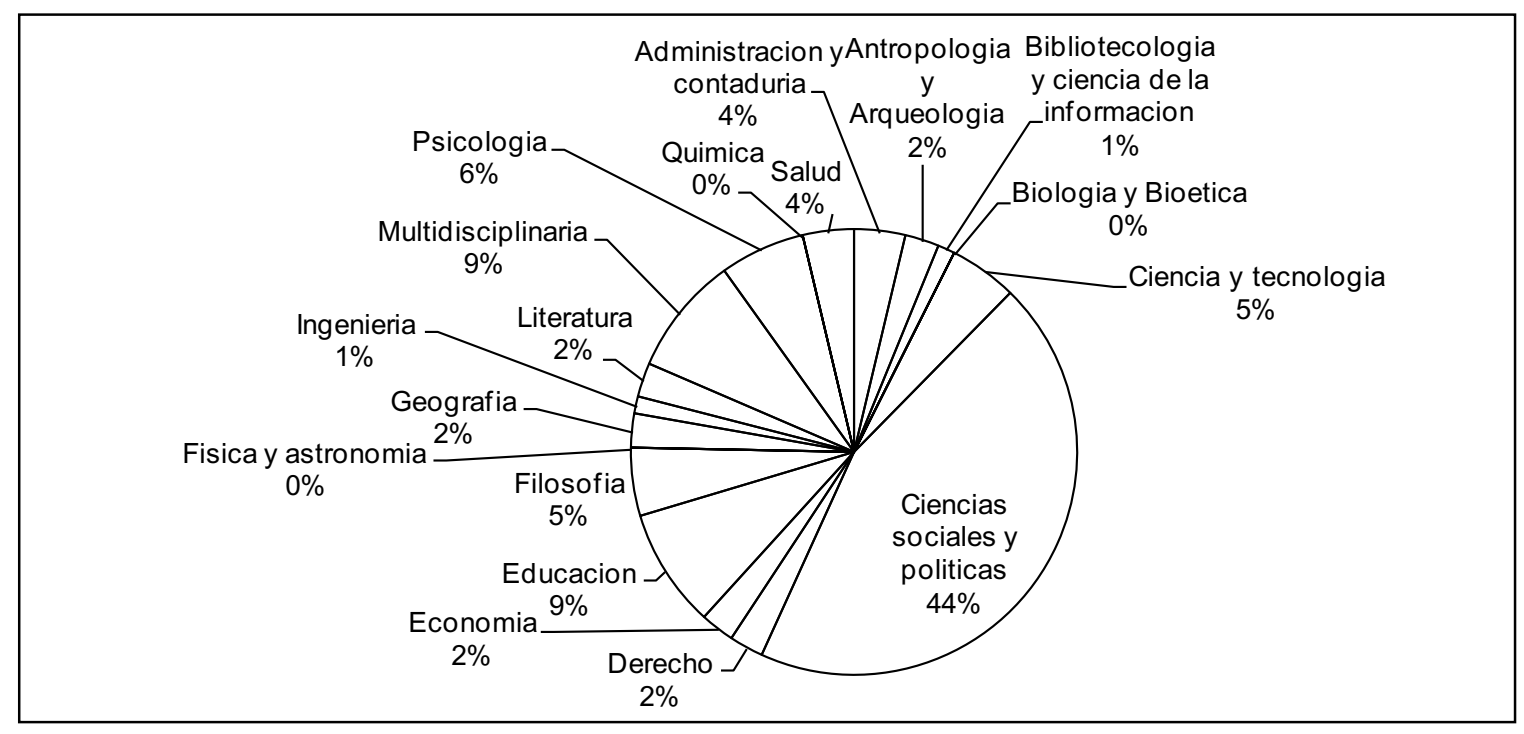




\section{GRÁFICO 12. DiSTRIBUCIÓN DE ARTÍCULOS POR TEMA}

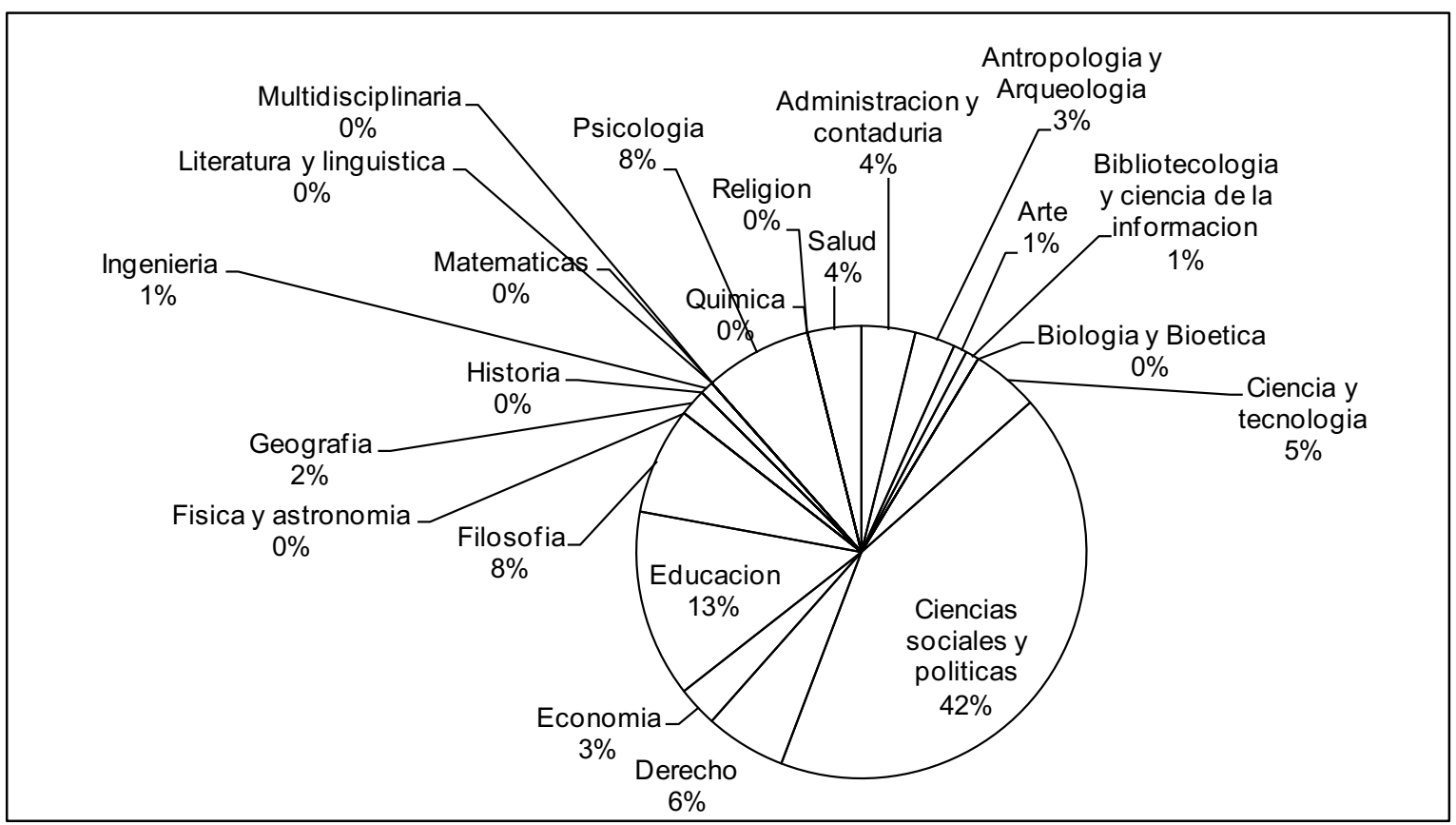

\section{GRÁFICO 13. DISTRIBUCIÓN DE KEYWORDS}

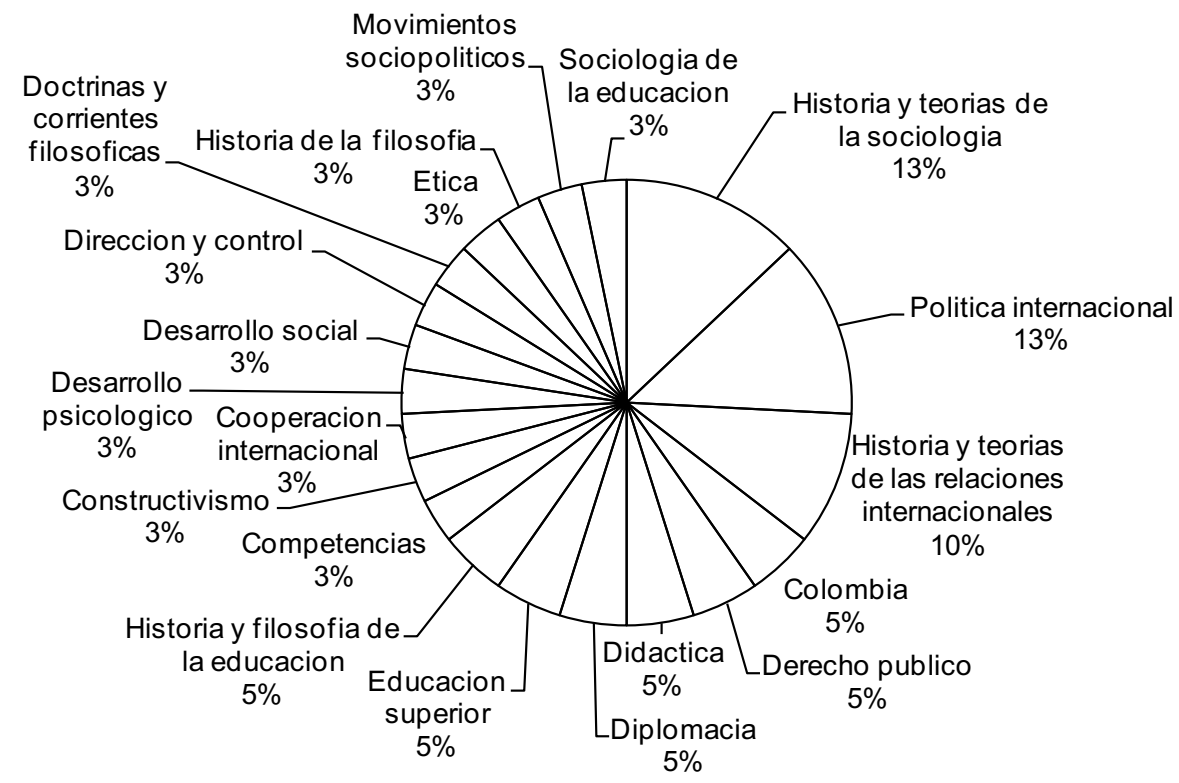


GRÁFICO 14. REFERENCIAS A AUTORES

\begin{tabular}{|c|c|c|c|c|c|c|}
\hline & 0 & 5 & 10 & 15 & 20 & 25 \\
\hline castorina ja & & & 1 & & & \\
\hline $\begin{array}{l}\text { latour } \mathrm{D} \\
\text { feenberg }\end{array}$ & & & & & & \\
\hline piaget $j$ & & 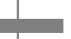 & & & & \\
\hline maturana $\mathrm{h}$ & & 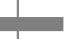 & & & & \\
\hline wendt a & & 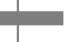 & & & & \\
\hline di meo $\mathrm{g}$ & & 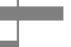 & & & & \\
\hline $\begin{array}{r}\text { ferreiro e } \\
\text { bijker w }\end{array}$ & & & & & & \\
\hline freire $p$ & 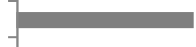 & & & & & \\
\hline $\begin{array}{r}\text { luhmann } n \\
\text { morin } e\end{array}$ & & & & & & \\
\hline callon $\mathrm{m}$ & & & & & & \\
\hline $\begin{array}{r}\text { ellul } j \\
\text { escobar } \mathbf{a}\end{array}$ & & & & & & \\
\hline hiernaux $\mathrm{d}$ & & & & & & \\
\hline perelman $f$ & & & & & & \\
\hline ruggie $\mathrm{j}$ & 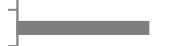 & & & & & \\
\hline berger $p$ & 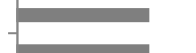 & & & & & \\
\hline $\begin{array}{r}\text { habermas } \mathrm{J} \\
\text { hacking } \mathrm{i}\end{array}$ & 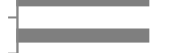 & & & & & \\
\hline kuhn $\mathrm{t}$ & & & & & & \\
\hline $\begin{array}{r}\text { bateson } \mathrm{g} \\
\text { bunge } \mathrm{m}\end{array}$ & & & & & & \\
\hline buttimer a & & & & & & \\
\hline garcia canclini n & ש & & & & & \\
\hline $\begin{array}{r}\text { marcuse } \mathrm{n} \\
\text { popper } \mathrm{k}\end{array}$ & 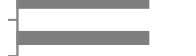 & & & & & \\
\hline
\end{tabular}

\section{CONCLUSIONES}

Nos propusimos observar al constructivismo a través de las publicaciones científicas que lo tratan en distintos campos del conocimiento. Comparando las publicaciones, delimitamos 3 grupos en los que el constructivismo reviste un 'uso particular'. En dichos usos varían los problemas y las preguntas, así como la inserción en ciertos campos disciplinares y temáticos. Si bien se mantienen algunas referencias generales que hacen del constructivismo un conglomerado general identificable, también difieren los programas constructivistas particulares y las corrientes noconstructivistas que se consideran para el diálogo.

Partimos el trabajo bajo el supuesto de la heterogeneidad del constructivismo. Nuestro análisis permite ahora distinguir dos tipos de heterogeneidad. En primer lugar, el constructivismo aparece como un discurso pluridisciplinario en el que distintas comunidades particulares le dan un sentido propio en vistas del uso que adquiere para sus problemáticas. Así, por ejemplo, no es el mismo recurso que se hace al constructivismo cuando se quiere fundamentar una práctica pedagógica que cuando se busca un análisis meta-teórico de un desarrollo constructivista particular. En segundo lugar, y al interior de estos grupos, el constructivismo es un 
Los usos del constructivismo en las publicaciones científicas de Latinoamérica

discurso muy genérico en el que conviven programas con tesis y supuestos diversos. Así, por ejemplo, en las publicaciones de pedagogía conviven programas constructivistas con supuestos filosóficos tanto realistas como idealistas que condicionan diferencialmente la forma en que se entiende el proceso de aprendizaje. En este trabajo nos hemos centrado en la primera forma de heterogeneidad para la que hemos buscado generar observables. La segunda forma de heterogeneidad tiene una cuantiosa literatura.

Podemos suponer que estas dos formas de heterogeneidad condicionan la integración del constructivismo como campo de comunicaciones. $\mathrm{Al}$ interior de cada uno de los grupos aquí distinguidos, dialogan comunidades que interpretan al constructivismo con sentidos "inconmensurables", si se nos permite llevar la noción de Kuhn muy lejos del campo de aplicación que este autor hubiera intencionado. No obstante, y como el mismo Kuhn ha señalado en su obra más tardía, la inconmensurabilidad no es necesariamente "absoluta": líneas de diálogo (si bien trabajoso) se pueden tender entre comunidades. El análisis comparado de conceptualizaciones y prácticas, y la discusión de convergencias y divergencias entre tesis y supuestos de distintos programas constructivistas puede ser útil para dinamizar los propios enfoques y/o permitir integraciones entre las áreas de latencia de cada programa.

Por otro lado, la distancia que separa a los grupos de publicaciones entre sí es mayor. En la medida en que los usos dados al constructivismo difieren, los sentidos del constructivismo que allí se discuten remiten a comunicaciones cuyo objeto no se intersecta. Esto no implica que las comunicaciones de un grupo no puedan referir a las de otro. Por ejemplo, este sería el caso de los artículos de corte más filosófico que analizan las diferentes corrientes del constructivismo pedagógico. Sin embargo, se debe advertir que esta referencia supone un cambio en el orden de la reflexión: un grupo se vuelve objeto del análisis de otro, o en otras palabras, las diferencias latentes en un grupo se vuelven observables para otro. Es de esperarse que esto se observe artículos que se guíen por más de un uso de los aquí reseñados. Conjeturar sobre la estructura de tales artículos y más aún sobre la organización de la comunicación que dan origen, nos ha sido vedado al optar por clasificaciones excluyentes con el objeto de lograr indicadores de publicación más claros. Futuros trabajos deberían ser capaces de lidiar con esta limitación.

El estudio aquí iniciado se puede continuar de diversas maneras. Por ejemplo, se puede ampliar la muestra para incluir términos afines (construcción social, construccionista, etc.); incluir otras bases de datos bibliográficas con indexación sobre distintas regiones e idiomas como REDALYC, PSYCINFO, o Web-of-Science (en esta última la búsqueda 'constructivism' devuelve más de 1000 registros, mucho más que la base que hemos construido); tratando con otras técnicas y métodos de exploración y minería de textos, como la teoría de grafos aplicada al análisis documental; y la consideración de la organización de la comunicación, 
como por ejemplo, a partir de relaciones de citado. Entendemos que el potencial valor de este enfoque no es otro que el de incorporar la observación empírica sobre las comunicaciones científicas a los más extendidos análisis epistemológicos sobre las tesis de las corrientes constructivistas. Creemos que así se podría lograr una visión más integrada sobre los diferentes sentidos y usos que adquiere el 'constructivismo'. $\mathrm{RM}$

\section{REFERENCIAS}

Andersen, N. (2003). Discursive Analytical Strategies.Understanding Foucault, Koselleck, Laclau, Luhmann. London: Policy Press.

Becerra, G. (2014). Ciencia y conocimiento en la teoría de los sistemas sociales de Niklas Luhmann. Sociología y tecnociencia. Revista digital de sociologia del sistema tecnocientífico, 4(2), 16-39.

Becerra, G., \& Castorina, J. A. (2016). Acerca de la noción de "marco epistémico" del constructivismo. Una comparación con la noción de "paradigma" de Kuhn. Revista Iberoamericana de Ciencia, Tecnología y Sociedad, 11(31), 9-28.

Bickhard, M. H. (1998). Constructivisms and Relativisms. A Shopper's Guide. In M. Matthews (Ed.), Constructivism in Science Education (pp. 99-112). Dordrecht: Springer.

Castellaro, M. A. (2012). Definiciones teóricas y áreas de investigación propuestas desde el constructivismo, en publicaciones latinoamericanas de psicología y educación presentes en la base de datos Redalyc. Liberabit. Revista de psicología, 18(2), 131146.

Castorina, J. A. (2007). El significado del análisis conceptual en psicología del desarrollo. In Epistemología e historia de la ciencia. Vol. 13 (pp. 132-138). Córdoba: Universidad Nacional de Córdoba.

Castorina, J. A. (2009). El constructivismo de inspiración piagetiana y el constructivismo radical. Un análisis crítico. En J. C. L. Narciandi, T. S. Criado, \& D. L. Gómez (Eds.), ¿Dónde reside la acción? Agencia, constructivismo y psicología (pp. 91-116). Murcia: Universidad de Murcia.

Coll, C. (1996). Constructivismo y educación escolar: ni hablamos siempre de lo mismo ni lo hacemos siempre desde la misma perspectiva epistemológica. Anuario de psicología, (69), 153-178.

Delval, J. (1997). Tesis sobre el constructivismo. En M. J. Rodrigo \& J. Arnay (Eds.), La construcción del conocimiento escolar (pp. 15-33). Barcelona: Paidós.

Delval, J. (2001). Hoy todos son constructivistas. Educere, 5(15), 353-359.

Feixas Viaplana, G., \& Villegas Besora, M. (2000). Constructivismo y psicoterapia. Biblioteca de psicología (Vol. 3 , rev). Bilbao: Desclée de Brouwer.

García, R. (2000). El conocimiento en construcción: De las formulaciones de Jean Piaget a la teoría de sistemas complejos. Barcelona: Gedisa.

Gibson, B., Gregory, J., \& Robinson, P. G. (2005). The Intersection between Systems Theory and Grounded Theory: The Emergence of the Grounded Systems Observer. Qualitative Sociology Review, 1(2), 3-21.

Glaser, B., \& Strauss, A. (1967). The Discovery of Grounded Theory: Strategies for Qualitative Research. Chicago: Aldine Publishing.

Guzzini, S. (2000). A Reconstruction of Constructivism in International Relations. European Journal of International Relations, 6(2), 147-182.

Hacking, I. (1999). The Social Construction of What? Massachusets: Harvard University Press.

Holstein, J., \& Gubrium, J. (2008). Handbook of Constructionist Research. New York: 
The Guilford Press.

Kuhn, T. S. (1970). The Structure of Scientific Revolutions ( $2^{\text {nd }}$ edition). Massachusets: Cambridge University Press.

Kukla, A. (2000). Social Constructivism and the Philosophy of Science. London: Routledge.

Lucio-Arias, D., \& Leydesdorff, L. (2009). The Dynamics of Exchanges and References among Scientific Texts, and the Autopoiesis of Discursive Knowledge. Journal of Informetrics, 3(3), 261-271.

Luhmann, N. (1997). La ciencia de la sociedad. México: Anthropos.

Munné, F. (1999). Constructivismo, construccionismo y complejidad: la debilidad de la crítica en la psicología construccional. Revista de psicología social, 14(2), 131-144.

Nola, R., \& Irzik, G. (2005). Philosophy, Science, Education, and Culture. London: Springer.

Noss, R., \& Clayson, J. (2009). Reconstructing Constructionism. Constructivist foundations, 10(3), 285-288.

Sánchez, J. C. (2009). Los límites del constructivismo. In J. C. Loredo, T. SánchezCriado, \& D. López Gomez (Eds.), ¿Dónde reside la acción? Agencia, constructivismo y psicología. (pp. 291-326). Madrid: UNED.

Stichweh, R. (2008). The Sociology of Scientific Disciplines: On the Genesis and Stability of the Disciplinary Structure of Modern Science. Science in Context, 5(1), 3-15.

SOBRE EL AUTOR

Gastón Becerra es Magíster en epistemología e historia de la ciencia por la Universidad Nacional de Tres de Febrero, Argentina. Licenciado en sociología por la Universidad de Buenos Aires. Actualmente se desempeña como académico de la Universidad de Buenos Aires, Argentina. Entre sus últimas publicaciones se encuentran: Una mirada social y política de la ciencia en la epistemología constructivista de Rolando García (2016), Connecting Second-Order Cybernetics' Revolution with Genetic Epistemology (2016), Acerca de la noción de 'marco epistémico' del constructivismo. Una comparación con la noción de 'paradigma' de Kuhn (2016).

\section{CONTACTO}

Facultad de Ciencias Sociales

Universidad de Buenos Aires

Marcelo T. de Alvear 2230 - C1122AAJ

Ciudad Autónoma de Buenos Aires

Argentina

gastonbecerra@sociales.uba.ar

Recibido: abril 2016

Aceptado: julio 2016 